\title{
Rescue Fly: An Essential
}

\author{
Baddam Sandeep Reddy \\ Student, Department of Mechanical Engineering \\ National Institute of Technology, Warangal \\ Warangal- 506004, Telangana, India
}

\begin{abstract}
The frequent words encountered in the current world are burglary, women abduction or some other crime when a person goes out alone. And there are multitude of cases that occur regularly. So, from the dire need of the society, a solution has been identified. The product described in this paper is designed keeping primary motto of solving these issues. In simple words, its mission is to make world free from monophobia when they are 'out'. This product is to be carried by a person whenever he/she goes out alone where it gets activated when the person is in danger. The product is a robotic fly which resembles dragonfly in shape and mosquito in action. This fly is governed by a microcontroller present in the case in which fly resides and no more human-control when it is in operation. The data required for human detection and others are fed into the case where it serves as repository. Upon opponent identification, it sedates them (one or many) and make them unconscious for certain amount of time thereby victim can escape. The communication protocol between them is chosen based on the less network join time and lower power consumption.
\end{abstract}

Keywords:- Maneuverability, Microcontroller, Zigbee communication.

\section{INTRODUCTION}

The major challenges faced in today's world when a person goes out alone are robbery, rape etc. To overcome these situations, a product is identified to solve the pressing need of the society. This product mainly consists of three components: 1. Hand band, 2. Case, 3. Robotic fly. Hand band measures pulse rate to detect whether a person in under threat or not and it can even be controlled manually if needed. Case, here not only holds robotic fly but also has several components for controlling and powering the fly. Robotic fly follows dragonfly's flying mechanism and has sedative storage in it. For vision, it uses ultra-small cameras during daylight and thermal imaging during night for human detection. As soon as the case receives signal from the band, it deploys robotic fly into operation and establishes Zigbee communication between them.

The fundamental concepts needed for rescue fly are flying mechanism, activation mechanism, communication, attacking mechanism, energy source and vision.

\section{FLYING MECHANISM}

Aeroplanes are heavier because they have separate mechanisms for flight and thrust i.e., they use turbojets for thrust and wings for flight. But, in case of birds, flight and thrust are accomplished by wings alone. This makes them lighter.
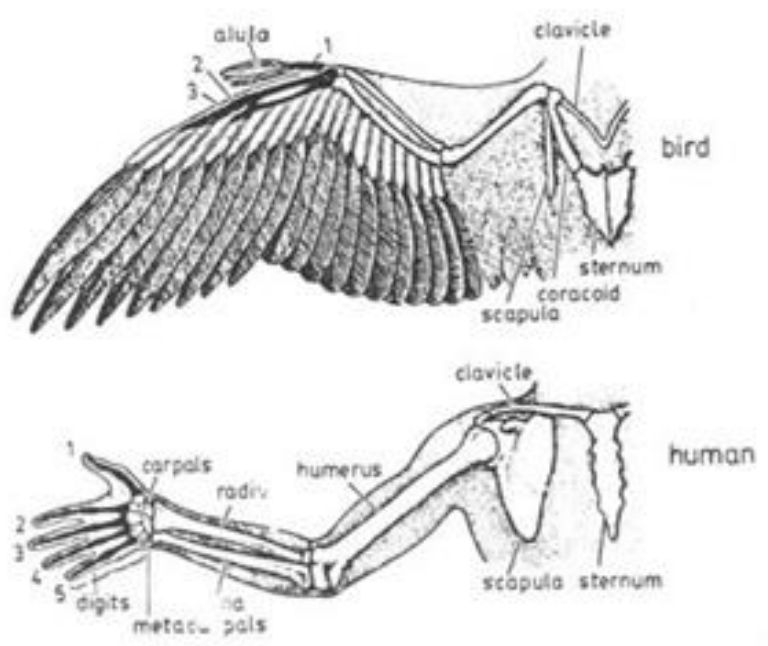

Fig 1

\section{Evolution: How analogy worked out?}

Over time in evolutionary process, the forelimbs were gradually transformed into wings. With the picture shown, one can find analogy between the bone structure of bird wing and human arm. 'Humerus', the upper arm is proportionately shorter, the wrist and palm bones are joined together for higher strength in supporting the primary flight feathers.

Essential elements for flight:

- A lightweight high-strength structure

- Wings for generating lift and forward thrust

- Energy source to provide the power

- A fast response flight control and navigation system

$>$ Briefflight mechanics of bird: are: Basic aerodynamic requirements for sustained flight

- enough lift to balance body weight,

- enough forward thrust to balance backward body drag 
As the wings of birds have to generate both lift and thrust, their motions, and forces on them which arise due to interaction with the air, are much more complex to analyse and quantify than on aircraft. The main aim in applying mechanical principles to bird flight is to relate the forces acting during flight to parameters such as wing dimensions and total weight.

The amount of lift (L) and drag (D) generated by the motion of a wing through the air depend upon five main factors:

- Wing shape (cross-section as well as planform)

- Angle of attack: Angle between the surface of wing and direction of air stream $(\alpha)$

- Area of the wing (S)

- Density of the air (r) and its kinematic viscosity (v)

- Velocity of the air stream relative to the wing (V)

Note: The planform area is the area of the wing as viewed from above the wing, looking along the "lift" direction

The relationship between these factors can be expressed as:

$\mathrm{L}=1 / 2 \mathrm{rV}^{2} \mathrm{SC}_{\mathrm{L}}, \quad \mathrm{D}=1 / 2 \mathrm{rV}^{2} \mathrm{SC}_{\mathrm{D}}$

where $C_{L}$ and $C_{D}$ are non-dimensional coefficients which depend upon the properties of the aerofoil section and Reynolds number (representing the ratio of the inertial to the viscous forces).

\section{Selection of flying mechanism:}

For this model, 'dragonfly' flying mechanism is preferred because of the following factors:

- High maneuverability

- Greater stabilized trajectory

The main reason behind its maneuverability is the presence of 4 wings. They enable it to quickly change direction, slow down, or accelerate extremely rapidly. The dragonfly uses both pair of wings for greater acceleration and directional changes. It could also conserve energy while hovering by flapping the wings at the same rate but out of phase (Out of phase is maintaining an angle of $180^{\circ}$ between front pair and rear pair).

The difference between the trajectory accomplished by a great skua (very large bird), a seagull (large bird) and a dragonfly.

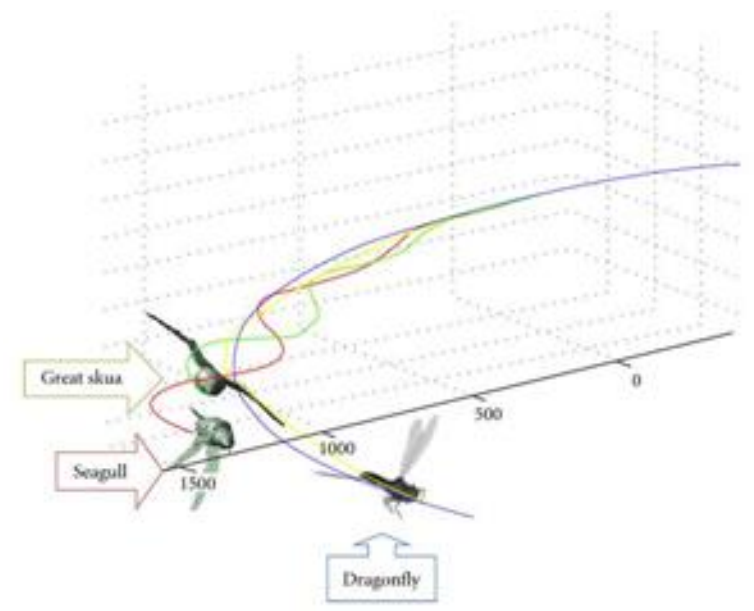

Fig 2

Image Source:

Micael S. Couceiro, N. M. Fonseca Ferreira, and J. A. Tenreiro Machado, Modelling and Control of a DragonflyLike Robot

The mechanism of flying for dragonfly is entirely different from that of two-winged insects. It's quite complex for analysing the fluid flow. In simple words, it can be understood as counter-stroking in most of the cases. At a stage during flight, front pair of wings flaps down leading to formation of vortex thereby a high air flow rate occurs on the top of rear pair of wings which increases lift (as in case of aerofoil). An almost steady lift is created making different states, downstroke and upstroke indistinguishable.

\section{Turbulence vs Dragonfly}

In case of aeroplanes, they make use of aerofoil shaped wings to create more flow on the top compared to bottom so that lift occurs because of Bernoulli's principle. Here, they need a smooth flow of air above and below the wings. A small turbulence can be disastrous. But, that's not the case for dragonfly where it augments lift by creating turbulence. There are many other flying organisms which use the same principle of vortex formation but dragonfly is a basic version of it.

Wing loading:

$\mathrm{W}=$ Weight supported by wings

$\mathrm{S}=$ Surface area of wings

Then, W/S is termed as 'wing loading factor'

\section{ACTIVATION MECHANISM}

In this proposed model, Hand band which is fastened on wrist activates microcontroller. Microcontroller further activates Rescue fly which performs its duty. Microcontroller can be activated through two modes. One is manual mode and the other, automatic mode. 
In automatic mode, band uses light absorbing property of haemoglobin for measuring pulse rate. Band has optical heart rate sensor inbuilt in it. The sensor has LED which projects green light on the skin and has photodetector which measures quantity of reflected light. The light that is not absorbed by the tissue beneath the skin is reflected back to the photodetector which generates electric signal, then converts from analog to digital signal. Eventually, it measures the changes to calculate pulse rate. If pulse rate crosses a particular threshold limit, then sends a signal to microcontroller thereby fly gets activated.
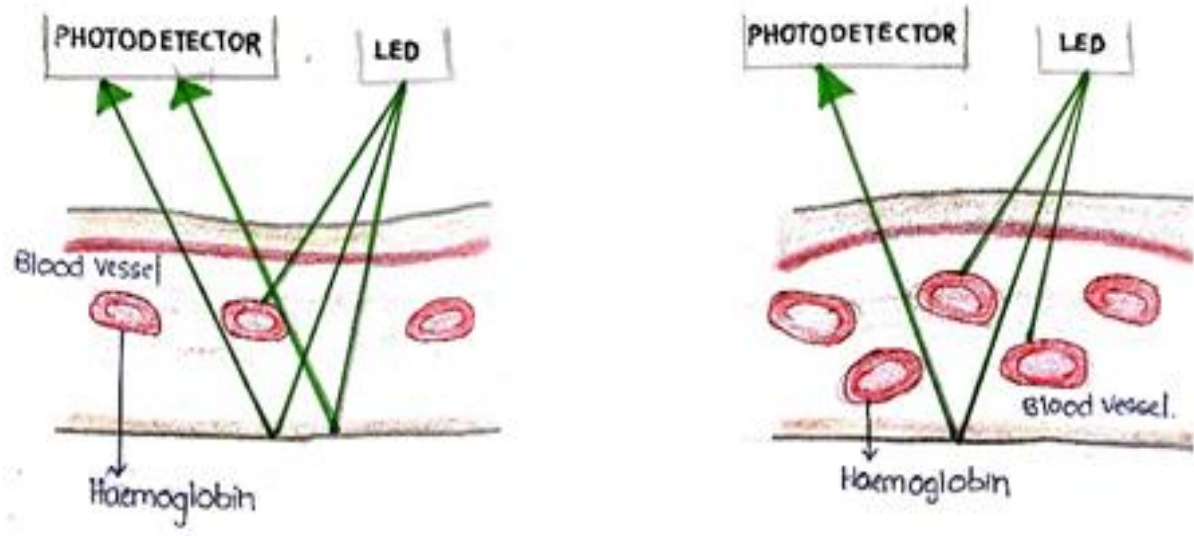

Fig 3:- showing contraction and expansion of blood vessel

In case of contraction, less amount of RBC (has haemoglobin inside it) enter the blood vessel, which results more amount of reflected light whereas in expansion more amount of RBC results less amount of reflected light.

\section{COMMUNICATION}

The devices here communicate each other using Zigbee protocol as shown in architecture. The reasons behind the selection are its features which perfectly accomplish the job. Zigbee standard is specially built for control and sensor networks. Since Wi- Fi may not be possible at every nook and corner, therefore Zigbee serves the purpose.
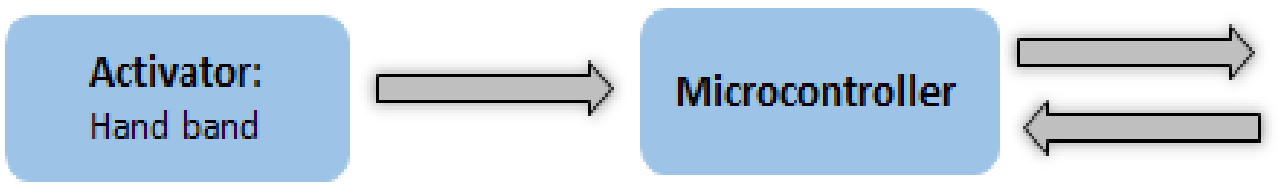

\section{Performer:}

Rescue fly

Fig 4:- Architecture of communication for the model

\section{Features of Zigbee:}

- Lower power consumption

- Network range is upto $100 \mathrm{~m}$

- Network join time: upto 30 millisec (for Wi- Fi: upto 3 sec and Bluetooth: upto $10 \mathrm{sec}$ )

- Lower data rates

\section{Why not Bluetooth?}

Zigbee aims at automation whereas Bluetooth aims at connectivity of mobile devices in the vicinity. Connectivity range for Zigbee is 3 times more compared to that of Bluetooth. Zigbee consumes low power, uses lower data transfer rates while Bluetooth consumes high power, uses higher data transfer rates. Moreover, network join time for Zigbee is very less compared to that of Bluetooth which differentiates a lot.

\section{ATTACKING MECHANISM}

As of now there are no drugs that can cause instant unconsciousness because any drug which is injected has to travel from the given site to brain. So, there is certain amount of time before it shows its effect and this cannot be avoided but may overcome in near future. Drugs like flunitrazepam and ketamine may not be instant but cause rapid unconsciousness. These are not fatal in low dosages.

Through the vision of Fly, this controller detects humans through real-time human detection algorithms which it got already pre-trained. Fly carries one of the mentioned sedatives in its repository and injects the opponent upon receiving signal from controller. 
As fly gets signal from its microcontroller, it leaps into operation. By detecting opponent, it attacks by injecting sedative through port $\mathrm{B}$. The beauty of this mechanism lies in the method of injecting as there are no separate processes for inserting and releasing the sedative. This reduces the time of injection or simply it is 'rapid injection'.
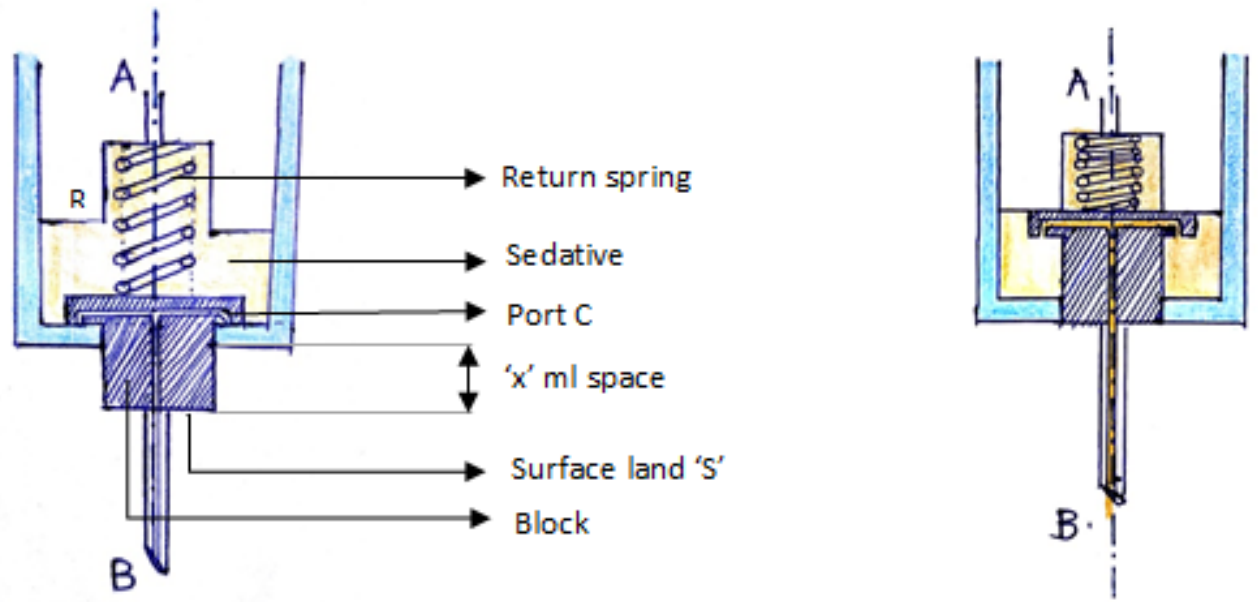

Fig 5:- showing injection mechanism at the start and end of injection process

\section{Working of injection mechanism:}

Once the opponent is detected, Fly inserts needle on exposed surface of skin and rests on land ' $\mathrm{S}$ '. This land ' $\mathrm{S}$ ' goes on moving upon insertion until it reaches rigid end ' $\mathrm{R}$ '. In this process, the block pushes spring behind it forcing the sedative into its ports ' $\mathrm{C}$ '. The amount of sedative injected is the amount of space the block displaced which is ' $\mathrm{x}$ ' $\mathrm{ml}$ space. Therefore, the volume of injection is shown as ' $\mathrm{x}$ ' $\mathrm{ml}$ space. And this ' $\mathrm{x}$ ' $\mathrm{ml}$ space depends on type of sedative.

After the injection process is done, the block returns to its original position with the aid of return spring thereby refilling the sedative space through port $\mathrm{A}$.

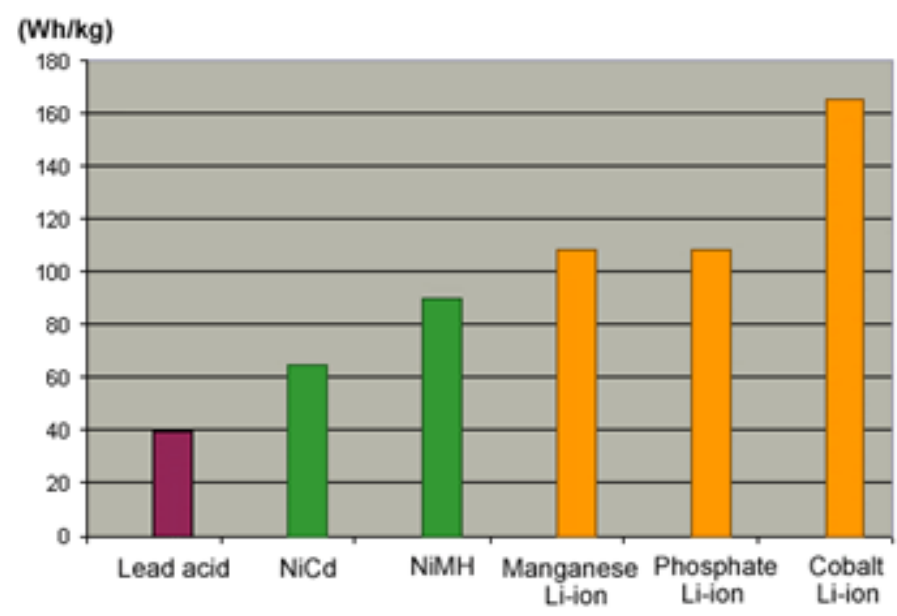

Fig 6

Image Source:

https://www.techsciresearch.com/blog/manufacturinglithium-ion-batteries/29.html

\section{ENERGY SOURCE}

In this model, the primary requirement for battery is its energy density. Thanks to Stanley Whittingham, John B. Goodenough and Akira Yoshino for their great contribution in the field of Lithium-ion batteries. Compared to all batteries present in the current domain, Cobalt lithium-ion battery has highest energy density of approximately 165 $\mathrm{Wh} / \mathrm{Kg}$. The pros and cons of this battery are:

\section{Advantages:}

- High energy density (165 Wh/kg)

- Lighter design

- Low self-discharge and longer shelf life

- Lower memory effect

- Quick charging

$>$ Disadvantages:

- Relatively short life span (among Li-ion's)

- Low thermal stability

- Limited load capabilities

\section{VISION}

The proposed system uses camera mounted on fly for human detection during daylight and thermal imaging during night. For identification, human detection and motion analysis algorithms run inside it as it captures the scene. This algorithm searches for humans in the image by matching its edge features to a database of templates of human images. Since fly is airborne, it further needs stabilization algorithm to run simultaneously. During nights, thermal imaging concept is used where it projects near infrared band of infrared light which ranges from 0.75 to $1.4 \mu \mathrm{m}$. 

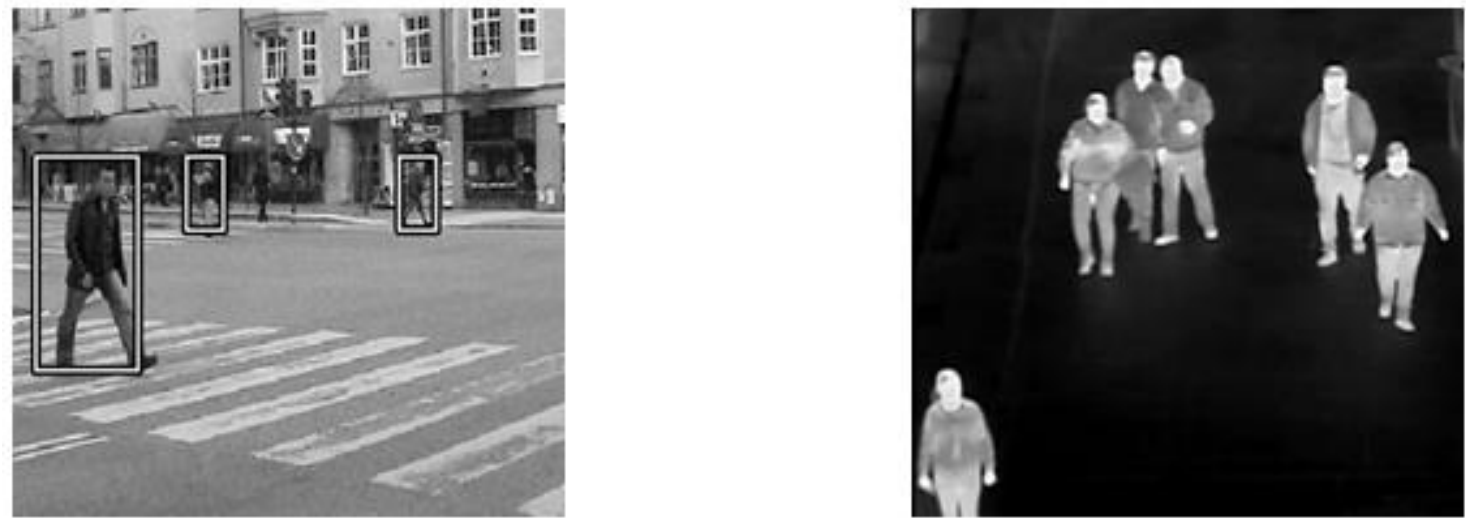

Fig 7:- Human detection: a) during day, b) during night

Image source:

a) https://www.indiamart.com/proddetail/human-detection-research-14267099973.html

b) hpthermalcamera.com

\section{CONCLUSION}

With a basic intention of rescuing people from danger, this product is put forth. Therefore, a myriad of cases can be eliminated in the near future. Moreover, this product can be integrated with automobiles thereby it cannot be considered as a separate device. Finally, this product needs a lot of research and development to bring into the market with the desired features.

Now a question may arise: Who is going to carry this product? Small reasoning for them. People carry a mobile phone and some bulky gadgets with a specific purpose. But one can decide whether this product's purpose is essential or not. Question of value comes next.

\section{REFERENCES}

[1]. Micael S. Couceiro, N. M. Fonseca Ferreira, and J. A. Tenreiro Machado, Modelling and Control of a Dragonfly-Like Robot

[2]. D. Gavrila. Pedestrian detection from a moving vehicle. In ECCV '00: Proceedings of the 6th European Conference on Computer Vision-Part II, pages 37-49, London, UK, 2000. Springer-Verlag.

[3]. Mohamed Hussein, Wael Abd-Almageed, Yang Ran, Larry Davis, Real-Time Human Detection, Tracking, and Verification in Uncontrolled Camera Motion Environments.

[4]. Joanna Tong \& Adele Schwab, The flight of birds

[5]. S Dhawan, Department of Space, Bird flight

[6]. http://www.robaid.com/bionics/researchers-observeflying-insects-to-create-smaller-flying-machines.htm

[7]. https://blogs.bu.edu/biolocomotion/2011/10/17/theastonishing-agility-of-the-dragonfly/

[8]. https://global.epson.com/innovation/core_technology/ wearable/vital_sensing.html 